\title{
ABELIAN THEOREM FOR THE STIELTJES TRANSFORM OF DISTRIBUTIONS
}

\author{
BOGOLJUB STANKOVIĆ \\ Institute of Mathematics \\ University of Novi Sad \\ Yugoslavia
}

(Received February 13, 1989 and in revised form March 15, 1989)

\begin{abstract}
First, a definition is given of the Stieltjes transform of distributions which contains some well-known. Then, a structural theorem for distributions having S-asymptotic is proved. This makes possible to prove two theorems of the Abelian type valued for Stieltjes transforms of distributions given in different ways.
\end{abstract}

KEYS WORDS AND PHRASES. Stieltjes transform, S-asymptotic, Abelian type theorem, distribution, generalized function.

1980 AMS SUBJECT CLASSIFICATION CODE. 46F 12.

1. INTRODUCTION.

It is possible to define the Stieltjes transform of distributions in various ways. One of them is the so-called direct approach in which we construct a basic space $A \supset 0$ of smooth functions to which the set $\left\{(s+t)^{-r-1}, \operatorname{Im} s \neq 0, r \geq 0\right\}$ belongs. Then, we define a transform for $T$, belonging to the dual space $A^{\prime}$, by the expresion $<T(t)$, $(s+t)^{-r-1}>$. In this paper we shall deal only with such definitions. If the constructed space $A^{\prime}$ is such that $T \in A^{\prime}$ has the support belonging to $[0, \infty)$, we have the classical Stieltjes transform, extended to generalized functions in one dimension.

We shall give a definition of the Stieltjes transform in meny-dimensional case not only to have a new generalisation of the Stieltjes transform, but to prove Abelian type theorems valued for the Stieltjes transforms given in different ways.

2. NOTATIONS AND DEFINITIONS.

$N$ is the set of natural numbers, $N_{0}=N \cup\{0\}$. If $a, b \in R^{n}, z \in C^{n}, z=\left(x_{1}+i y_{1}, \ldots\right.$ $\left.\ldots, x_{n}+i y_{n}\right)$ and $e=(1, \ldots, 1)$, then: $(a \cdot b)=\sum_{i=1}^{n} a_{i} b_{i} ; z^{a}=\prod_{i=1}^{n}\left(x_{i}+i y_{i}\right)^{a_{i}} ;\left|z^{a}\right|=\prod_{i=1}^{n} \mid x_{i}+$ $+\left.i y_{i}\right|^{a_{i}} ; z^{e}=\prod_{i=1}^{n} z_{i} ;\|a\|^{2}=(a \cdot a) ; a \geq 0$ means $a_{i} \geq 0$ for all $i=1, \ldots, n$.

$\Gamma$ will be a convex acute cone with vertex at zero; $B(0, r)$ the closed,ball with the center at zero and with radius $r>0 ; S(0,1)$ the unit sphere, all in $R^{n}$. ${ }^{\circ}$ is the interior of $\Gamma$. 
DEFINITION 1. Let $h_{1}, h_{2} \in \Gamma$. We say that $h_{1} \geq h_{2}$ in $\Gamma$ if $h_{1} \in h_{2}+\Gamma$. For a complex valued function $G(h), h \in \Gamma, \lim G(h)=A \in C, h \in \Gamma, h \rightarrow \infty$ if for every $\varepsilon>0$ there exists $h(\varepsilon) \in \Gamma$ such that $|G(h)-A|<\varepsilon$ when $h \geq h(\varepsilon)$ in $\Gamma$.

If $\stackrel{\circ}{\Gamma} \neq \emptyset$, we can prove the following Lemma:

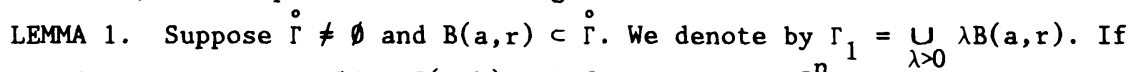
$\lim _{h \in \Gamma, h \rightarrow \infty} G(h)=A$, then $\lim _{h \in \Gamma} G(x+h)=A$ for every $x \in R^{n}$.

PROOF. By Definition 1 for every $\varepsilon>0$ there exists $h(\varepsilon)$ such that $|G(h)-A|<\varepsilon$, $h \in h(\varepsilon)+\Gamma$. Now $x-h(\varepsilon)+\beta_{0} a \in B\left(\beta_{0} a, \beta_{0} r\right)$ if $\beta_{0} \geqq\|x-h(\varepsilon)\| / r, \beta_{0}$ depends on $\varepsilon$. Hence $x-h(\varepsilon)+\beta_{0} a \in \Gamma_{1} \subset \Gamma$. Since $\Gamma$ is a convex cone, $x-h /(\varepsilon)+\beta_{0} a+\Gamma_{1} \subset \Gamma+\Gamma \Gamma_{1}$ $c \Gamma$ and $x+\beta_{0} a+\Gamma_{1} \subset h(\varepsilon)+\Gamma$. It follows that $|G(x+h)-A|<\varepsilon$ for $h \in \beta_{0} a+\Gamma_{1} .^{-}$

REMARK. In Lemma 1 we can choose the ball $\mathrm{B}(\mathrm{a}, \mathrm{r})$ in such a way that the distance between $B(a, r)$ and any coordinate axis $X_{i}, i=1, \ldots, n$, in $R^{n}$ is positive, $d\left(B(a, r), X_{i}\right)$ $\geq \alpha>0$. Then $\left|a_{i}\right|-r \geq \alpha>0, i=1, \ldots, n$. We denote by $\Gamma_{2}=\underset{\lambda>0}{\cup} \lambda B(a, r)$, for such constructed ball $\mathrm{B}(\mathrm{a}, \mathrm{r})$. If $\mathrm{u} \in \mathrm{h}_{0}+\Gamma_{2}$ for $a h_{0} \in \Gamma_{2}$, then $u=\lambda_{0} a+\lambda_{0} z+\lambda a+\lambda y$, where $z, y \in B(0, r) ; \lambda_{0}, \lambda>0$. It is easy to see that $\left|u_{i}\right| \geq\left(\lambda+\lambda_{0}\right)\left(\left|a_{i}\right|-r\right) \geq(\lambda+$ $\left.+\lambda_{0}\right) \alpha$ and for any $M>0$ we can find $h_{0}$ with the property $\left|u_{i}\right| \geq M$. Hence, if $h \rightarrow \infty$, $h \in \Gamma_{2}$, then $\left|h_{i}\right| \rightarrow \infty, i=1, \ldots, n$.

By $P$ we denote the set of all the real and positive functions $c$. Notations for the spaces of distributions are as in the book of L. Schwartz [1].

DEFINITION 2. A distribution $T \in D^{\prime}$ has the $S$-asymptotic in $\Gamma$ related to the function $c \in P$ and with the limit $U \in D^{\prime}$, if the following limit exists

$$
\lim _{h \in \Gamma, h \rightarrow \infty}\langle T(x+h) / c(h), \phi(x)\rangle=\langle U, \phi\rangle, \phi \in 0 .
$$

Then we write $T(x+h) \& c(h) \cdot U(x), h \in \Gamma$ and we say that $T$ has the S-asymptotic in $D^{\prime}$ (Pilipović and Stanković [2] and Stanković [3]).

We shall use the same definition for a $T \in B^{\prime} \equiv D^{\prime}\left(L^{\infty}\right)$ and $\phi \in O\left(L^{2}\right)$ stressing that $\mathrm{T}$ has the $\mathrm{S}$-asymptotic in $B^{\prime}$.

If the interior of the cone $\Gamma$ is not empty, $\stackrel{\circ}{\Gamma} \neq \emptyset$, then we can give the analytical expression of the limit distribution $U$ :

Suppose that $T \in O^{\prime}$ and has the $S$-asymptotic with the limit $U \neq 0$. Then there exists a $\phi_{0} \in D$ such that $\left\langle U, \phi_{0}\right\rangle \neq 0$. For this $\phi_{0}$ and $t \in R^{n}$, using Lemma 1 , we have

$$
\left.\lim _{h \in \Gamma_{2}, h \rightarrow \infty} \frac{c(h+t)}{c(h)}<\frac{T(x+(h+t))}{c(h+t)}, \phi_{0}(x)\right\rangle=\lim _{h \in \Gamma_{2}, h \rightarrow \infty}\left\langle\frac{T((x+t)+h)}{c(h)}, \phi_{0}(x)\right\rangle .
$$

From trhis relation it follows the existence of the following limit

$$
\lim _{h \in \Gamma_{2}, h \rightarrow \infty} c(h+t) / c(h)=d(t), t \in R^{n}
$$

and that $U$ satisfies the equation

$$
\mathrm{d}(\mathrm{t})\left\langle\mathrm{U}, \phi_{0}\right\rangle=\left\langle\mathrm{U}(\mathrm{x}+\mathrm{t}), \phi_{0}(\mathrm{x})\right\rangle, \mathrm{d}(0)=1, \mathrm{t} \in \mathrm{R}^{\mathrm{n}} \text {. }
$$

Since $U$, as a distribution, has all the derivatives, it follows from relation (2.4) that:

$$
\begin{gathered}
{\left[d\left(t+\Delta t_{1}\right)-d(t)\right]\left\langle U, \phi_{0}\right\rangle=\left\langle U\left(x+t+\Delta t_{1}\right)-U(x+t), \phi_{0}(x)\right\rangle} \\
=\left[d\left(\Delta t_{1}\right)-d(0)\right] d(t)\left\langle U, \phi_{0}\right\rangle, d(0)=1 .
\end{gathered}
$$


It is now easy to prove that (Pilipovic and Stankovic [2]):

$$
d(t)=\exp (a \cdot t) \text { and } U(t)=C \exp (a \cdot t), t \in R^{n}
$$

\section{STIELTJES TRANSFORM OF DISTRIBUTIONS}

In the following we shall use the well-known function $\eta_{\varepsilon} \in C^{\infty}, \omega>0$ (Vladimirov [4]):

where

$$
\eta_{\varepsilon}(x)=\int_{B(0,2 \omega)} q_{\omega}(x-t) d t, x \in R^{n}
$$

$$
q_{\omega}(x)=\left\{\begin{array}{ll}
D \omega^{-n} \exp \left(-\frac{\omega^{2}}{\omega^{2}-\|x\|^{2}}\right), & \|x\|<\omega \\
0 & \|x\| \geq \omega
\end{array} ; D \int_{R^{n}} q_{1}(t) d t=1 .\right.
$$

The function $\eta_{\omega}$, has the properties: $0 \leq \eta_{\omega}(x) \leqq 1, x \in R^{n} ; \eta_{\omega}(x)=1, x \in B(0, \omega)$; $\eta_{\omega}(x)=0,\|x\|>3 \omega ;\left|D^{k} \eta_{\omega}(x)\right| \leq C_{k} \omega^{-(k \cdot e)}, x \in R^{n}$. The constants $C_{k}$ do not depend on $\omega$.

DEFINITION 3. The Stieltjes transform of a distribution $T \in D^{\prime}$ ( $s_{\rho}$-transform) is defined by the limit

$$
\lim _{\omega \rightarrow \infty}\left\langle T(x), \eta_{\omega}(x)(s+x)^{-(\rho+e)}\right\rangle=S_{\rho}(T)(s), s \in(C \backslash R)^{n},
$$

if it exists for a $\rho \in R^{n}$. By e we denoted $e=(1, \ldots, 1)$.

REMARK. s can belong to a larger set, as well. This depends on the support of $T$. So $s_{\rho}(\delta)(s)=s^{-(\rho+e)}, s^{-} \neq 0$.

We shall give a relation between Definition 3 of the Stieltjes transform and definitions of some other authors frequently used in many papers. All of them are in one dimension. Let us start with the classical definition.

If $T$ is defined by the function $f$, supp $f \subset[0, \infty)$, Definition 3 gives the classical Stieltjes transform, if it exists. Let $s \in(C \backslash(-\infty, 0])$, then

$$
\begin{aligned}
S_{\rho}(f)(s)=\lim _{\omega \rightarrow \infty} \int_{0}^{3 \omega} f(t) \eta_{\omega}(t)(s+t)^{-(\rho+1)} d t=\lim _{\omega \rightarrow \infty} \int_{0}^{\omega} f(t)(s+t)^{-(\rho+1)} d t+ \\
\quad+\lim _{\omega \rightarrow \infty} \int_{\omega}^{3 \omega} f(t) \eta_{\omega}(t)(s+t)^{-(\rho+1)} d t .
\end{aligned}
$$

We have only to prove that:

$$
\lim _{\omega \rightarrow \infty} \int_{\omega}^{3 \omega} f(t) \eta_{\omega}(t)(s+t)^{-(\rho+1)} d t=0, \quad s \in(C \backslash(-\infty, 0])
$$

when the classical Stieltjes transform exists.

Since for $\omega \leqq x \leqq 3 \omega$

$$
\eta_{\omega}(x)=\int_{-2 \omega}^{2 \omega} q_{\omega}(x-t) d t=\int_{(x-2 \omega) / \omega}^{1} q_{1}(y) d y ;
$$

the function $\eta_{\omega}(x)$ is positive and monotone decreasing in this interval. By the mean value theorem, there exists a $\xi, 0<\xi<2$ such that

$$
\int_{\omega}^{3 \omega} \eta_{\omega}(t) \operatorname{Re}\left[f(t)(s+t)^{-(\rho+1)}\right] d t=\eta_{\omega}(\omega) \int_{\omega}^{\omega+\xi \omega} \operatorname{Re}\left[f(t)(s+t)^{-(\rho+1)}\right] d t .
$$

The last integral tends to zero when $\omega \rightarrow \infty$, because we suposed that the Stieltjes transform of function $f$ exists. We have the same situation with the imaginary part of the 
integral from relation (3.5).

Lavoine and Misra [5-6] defined the Stieltjes transform of distributions belonging to a subset $J^{\prime}(r), \operatorname{Re} r>-1$ of $D^{\prime}$, which is used in many papers. A distribution $T$ belongs to $J^{\prime}(r)$ if and only if there exist $m \in N$ such that $T=D^{m} G$, where $G$ is a locally integrable function having a support in $[0, \infty)$ and $G(x)=o\left(x^{r+m-\alpha}\right), \alpha>0$. The Stieltjes transform of $T \in J^{\prime}(r)$ is, by these two authors:

$$
\mathrm{S}_{\mathbf{r}}(\mathrm{T})=(\mathrm{r}+1) \ldots(\mathrm{r}+\mathrm{m}) \int_{0}^{\infty} \mathrm{G}(\mathrm{t})(\mathrm{s}+\mathrm{t})^{-\mathrm{r}-\mathrm{m}-1} \mathrm{dt}, \quad s \in \mathcal{C} \backslash(-\infty, 0] .
$$

For a $T \in J^{\prime}(r), r=\rho$, relation (3.3) gives

$$
\begin{gathered}
S_{\rho}(T)(s)=\lim _{\omega \rightarrow \infty}\left\langle T(x), \eta_{\omega}(x)(s+x)^{-(\rho+1)}\right\rangle= \\
=(-1)^{m} \sum_{k=1}^{m}\left(\begin{array}{l}
m \\
k
\end{array}\right)(\rho+1) \ldots(\rho+m) \lim _{\omega \rightarrow \infty} \int_{0}^{\infty} \frac{G(x)}{(s+x)^{\rho+k+1}} D^{m-k} \eta_{\omega}(x) d x .
\end{gathered}
$$

We have two types of integrals

$$
\int_{0}^{\infty} \frac{G(x)}{(s+x)^{p+m+1}} \eta_{\omega}(x) d x \text { and } \int_{\omega}^{3 \omega} \frac{G(x)}{(s+x)^{p+k+1}} D^{m-k} \eta_{\omega}(x) d x, m-k \geq 1 .
$$

For the first one we proceed as in the case of the classical stieltjes integral. The second one tends to zero when $\omega \rightarrow \infty$. We have only to start from

$$
\int_{\omega}^{3 \omega} \frac{G(x)}{(s+x)^{p+m+1}}(s+x)^{m-k} D^{m-k} \eta_{\omega}(x) d x
$$

and to use the integration by parts.

In the next cases the authors followed the construction of the Stieltjes transform as we mentioned in the Introduction. The basic spaces A are topological vector spaces of complex valued smooth functions. The space $A$ contains $D$ and the topology of $D$ is stronger then that induced on it by $A$. The retstriction $T$ of an element $\bar{T} \in A^{\prime}$ to $D$ is in $D^{\prime}$. For $a \equiv \geq$ and $s$ belonging to a subset of $C,(s+t)^{-(\rho+e)}$ is in $A$. The Stieltjes transform of a $\bar{T} \in A^{\prime}$ is defined by $\left\langle\bar{T}(t),(s+t)^{-(\rho+e)}\right\rangle$.

In all the cases, we shall list, the family $\left\{(s+t)^{-(\rho+e)} \eta_{\omega}(t), \omega>0\right\}$ converges in $A$ to $(s+t)^{-(\rho+e)}$ when $\omega \rightarrow \infty$. Then for $a \bar{T} \in A^{\prime}$ and $T \in D^{\prime}$, we have:

$$
S_{\rho}(T)(s)=\lim _{\omega \rightarrow \infty}\left\langle\bar{T}(x), \eta_{\omega}(x)(s+x)^{-(\rho+e)}\right\rangle=\left\langle\bar{T}(x),(s+x)^{-(\rho+e)}\right\rangle
$$

By Zemanian [7] it is $\rho=0$ and the basic space $A$ is $J_{c, d}=\left\{\psi \in C_{(0, \infty)}^{\infty}, P_{c, d, k}(\psi)=\right.$ $\left.\sup _{0<t<\infty} x_{c, d}(t)|(t D) \sqrt[k]{t} \psi(t)|<\infty, k \in N_{0}\right\}$, where $x_{c, d}(t)=t^{c}, 1 \leqq t<\infty ; x_{c, d}(t)=t d$, $0<t<1$ and $c<1 / 2, d>-1 / 2$. The topology of $J_{c, d}$ is defined by the seminorms $P_{c, d, k}$ $k \in N_{0}$.

By Pandey [8] the basic space $A$ is $S_{\alpha}=\left\{\phi \in C_{(0, \infty)}^{\infty}, \gamma_{k}(\phi)=\sup \left(1+x^{\alpha}\right) \mid(x D)^{k} \phi(x)\right) \mid$ $\left.<\infty, k \in N_{0}\right\}$. The topology in $S_{\alpha}$ is defined by the seminorms $\gamma_{k}, k \in N_{0}$. The function $(s+t)^{-(\rho+1)} \in S_{\alpha}$ for $\alpha \leqq \rho+1$, but the family $\left\{(s+t)^{-(\rho+1)} \eta_{\omega}(t), \omega>0\right\}$ tends to $(s+t)^{-(\rho+1)}$ in $S_{\alpha}$ for $\alpha<\rho+1$ when $\omega \rightarrow \infty$.

Bremermann [9] introduced the basic space $0_{\alpha}=\left\{\zeta \in E(R), \zeta^{(k)}(t)=0\left(t^{\alpha}\right), k \in N_{0}\right\}$. The topology of $0_{\alpha}$ is that induced by $E$. He treated the case $\rho=0$, Im $s \neq 0$ and $\alpha \geq-1$. For the Stieltjes transform of distributions in many dimensional case see also [10]. 


\section{DISTRIBUTIONS HAVING THE S-ASYMPTOTIC}

We shall prove a structural theorem for the distributions having the S-asymptotic in a cone $\Gamma$ with the nonempty interior, $\stackrel{\Gamma}{\Gamma} \neq \emptyset$.

THEOREM 1. Suppose $T_{0} \in B^{\prime}$ and $T_{0}(x+h) \stackrel{s}{\sim} 1 \cdot U(x), h \in \Gamma$ in $D^{\prime}$, then

a) $\mathrm{U}=\mathrm{C}$;

b) $T_{0}=\sum_{i=0}^{2} \Delta \Delta^{i k} F_{i}$, where $F_{i}$ are continuous functions belonging to $L^{\infty}$;

c) For every $0 \leqq i \leqq 2$ functions $F_{i}(x+h)$ converge uniformly to a constant when $x$ belongs to a compact set $K$ and $h \in \Gamma, h \rightarrow \infty$;

d) $T_{0}$ has the S-asymptotic in $B^{\prime}$, as well, related to $c=1$ and with the limit $U=$ $=\mathrm{C}$ in the cone $\Gamma$.

PROOF. a) By relation (2.6) $U$ has to be a constant because $c=1$.

b) From the fact $T_{0} \in B^{\prime}$ it follows that $\left(\mathrm{T}_{0} * \hat{\zeta}\right) \in L^{-}$for every $\zeta \in D$ (Schwartz [1], II, p. 57) and the set of distributions $Q=\left\{T_{h} \equiv T_{0}(x+h), h \in R^{n}\right\}$ is weakly bounded and bounded in $D^{\prime}$.

In addition to 2 , we shall construct an other bounded set of distributions. We denote by $S=\left\{\psi \in 0,\|\psi\|_{L^{1}} \leq 1\right\}$. We have seen that for a fixed $\zeta \in D,\left(\mathrm{~T}_{0} * \bar{\zeta}\right) \in L^{\infty}$. Now, for every $\psi \in S$ :

$$
\begin{aligned}
\left|\left\langle\mathrm{T}_{0} * \hat{\psi}, \zeta\right\rangle\right| & =\left|\left\langle\mathrm{T}_{0} * \hat{\zeta}, \psi\right\rangle\right|=\left|\int_{R}\left(\mathrm{~T}_{0} * \hat{\zeta}\right)(\mathrm{t}) \psi(\mathrm{t}) \mathrm{d} t\right| \leq \\
& \leq\left\|\mathrm{T}_{0} * \hat{\zeta}\right\|_{L^{-}}\|\psi\|_{L^{1}} \cdot
\end{aligned}
$$

Hence the set of regular distributions, defined by the set of continuous functions $H=\left\{U_{\psi} \equiv T_{0} * \psi, \psi \in S\right\}$ is weakly bounded and bounded in $D^{\prime}$.

$A$ set $W^{\prime} \in D^{\prime}$ is bounded if and only if for every $\alpha \in D$ the set of functions $\{T * \alpha$, $\left.T \in W^{\prime}\right\}$ is bounded on every compact set $M$ belonging to $R^{n}$ (Schwartz [1], II, p. 50). Hence $\left\{T * \alpha, T \in W^{\prime}\right\}$ defines a bounded set of regular distributions. In such a way $\left\{\mathrm{T}_{\mathrm{h}} * \zeta, \mathrm{T}_{\mathrm{h}} \in 2\right\}$ and $\left\{\mathrm{U}_{\psi} * \zeta, \mathrm{U}_{\psi} \in H\right\}$ give two bounded sets of regular distributions. Now, for these two sets we can repeat twice a part of the proof of Theorem XXII from Schvartz [1], II, p. 51 .

We denote by $\Omega$ an open neighbourhood of zero in $R^{n}$ which is relatively compact in $R^{n}, c 1 \Omega=K$ a compact set. Then, by the mentioned part of the proof by Schwartz [1], there exist $m_{1} \geq 0$ and $m_{2} \geq 0$, such that the mappings $(\alpha, \beta) \rightarrow U_{\psi} *(\alpha * \beta)$ or $(\alpha, \beta) \rightarrow T_{h} *$ $*(\alpha * \beta)$ are equicontinuous and map $D_{\Omega}^{m_{1}} \times D_{\Omega}^{m_{1}}$ or $D_{\Omega}^{m_{2}} \times D_{\Omega}^{m_{2}}$ into $L_{B}^{\infty}$; B is the ball $B(0, r)$ where $r$ is a positive number. Hence, for every $x \in B$ and $h \in R^{n}$ the function $\left(T_{h} * \alpha * \beta\right)(x)=$ $\left(T_{0} * \alpha * \beta\right)(x+h)$ is continous.

Let $Z(0, \rho)$ be a ball in $L_{B}^{\infty}$, then there exists a neighbourhood $V_{1}\left(m_{1}, \varepsilon_{1} K_{1}\right)$ in $D_{\Omega}^{m_{1}}$, such that $U_{\psi} *(\alpha * \beta) \in Z(0, \rho)$ for $\alpha, \beta \in V_{1}\left(m_{1}, \varepsilon_{1}, K_{1}\right), U_{\psi} \in H$ and a neighbourhood $V_{2}\left(m_{2}, \varepsilon_{2}, K_{2}\right) \subset D_{\Omega}^{m_{2}}$, such that $T_{h} *(\alpha * \beta) \in Z(0, \rho)$ for $\alpha, \beta \in V_{2}\left(m_{2}, \varepsilon_{2}, K_{2}\right), T_{h} \in Q$. Let $K_{0}=K_{1} \cap K_{2}, \varepsilon_{0}=\min \left(\varepsilon_{1}, \varepsilon_{2}\right)$ and $m=\max \left(m_{1}, m_{2}\right)$. We shall now use relation $(V I, 6 ; 23)$ from Schwartz [1], II:

$$
T_{0}=\Delta^{2 k} *(\gamma E * \gamma E * T)-2 \cdot \Delta *(\gamma E * \xi * T)+(\xi * \xi * T),
$$

where $E$ is a solution of the iterated Laplace equation; $\Delta^{k} E=\delta ; \gamma, \xi \in D_{\Omega}$, suppr and supp $\xi$ belonging to $K_{0}=K_{1} \cap K_{2}$. We have only to choose the number $k$ large enough so 
that $\gamma E \in D_{\Omega}^{\mathrm{m}}$. Now, we can take: $F_{2}=\gamma E * \gamma E * T_{0} ; F_{1}=\gamma E * \xi * T_{0}$ and $F_{0}=\xi * \xi * T_{0}$. A11 of these functions are of the from: $F_{i}=T_{0} * \alpha_{i} * \beta_{i} ; \alpha_{i}, \beta_{i} \in V\left(m, \varepsilon_{0}^{\prime}, K_{0}\right), \varepsilon_{0}^{\prime}>0$.

We have to prove that $F_{i}$ have the properties given in Theorem 1 . For $\alpha_{i}, \beta_{i} c$ $U\left(\mathrm{~m}, \varepsilon_{0}^{\prime}, K_{0}\right)$ and $\psi \in S$

$$
\left|<\left(\mathrm{T}_{0} * \alpha_{i} * \beta_{i}\right), \psi>\right|=\left|\left[\left(\mathrm{T}_{0} * \hat{\psi}\right) *\left(\hat{\alpha}_{i} * \hat{\beta}_{i}\right)\right](0)\right| \leqq \rho\left(\varepsilon_{0}^{\prime} / \varepsilon_{0}\right)^{2} \equiv M
$$

Now let $\mu \neq 0$ be any element from $L^{1}$, then $\mu /\|\mu\|_{L^{1}} \in S$ and $\mid\left\langle\left(T_{0} *\left(\alpha_{i} * \beta_{i}\right)\right), \mu>\right| \leq$ $\leqq M\|\mu\|_{L^{1}}$ which proves that $T_{0} *\left(\alpha_{i} * \beta_{i}\right)$ belong to $L^{\infty}$. Since $F_{i}=T_{0} *\left(\alpha_{i} * \beta_{i}\right), \alpha_{i}, \beta_{i} \in$ $V\left(m, \varepsilon_{0}^{\prime}, K_{0}\right), F_{i}$ are continuous and belong to $L^{\infty}$.

c)We shall continue with investigations of the properties of $F_{i}$. By the properties of the convolution we have that $F_{i}(x+h)=F_{i} * \tau_{-h}=T_{0} *\left(\alpha_{i} * \beta_{i}\right) * \tau_{-h}=T_{h} *\left(\alpha_{i} * \beta_{i}\right)$ for $\alpha_{i}$, $\beta_{i} \in D_{\Omega}^{m}$, where $\tau_{-h}$ is the translation operator.

We have proved that the mappings $(\alpha, \beta) \rightarrow T_{h} * \alpha * \beta, T_{h} \in Q$, are equicontinuous and map $D_{\Omega}^{\mathrm{m}} \mathrm{x} D_{\Omega}^{\mathrm{m}}$ into $L_{B}^{\infty}$. $D$ is a dense subset od $D^{\mathrm{m}}, \mathrm{m} \geq 0$. We can construct a subset of $D_{K}$, c1 $\Omega=K$, which is dense in $0_{\Omega}^{m}$. Since $T_{h} *(\zeta * \psi) \rightarrow C * \zeta * \psi$ for $\zeta * \psi \in D_{\Omega} \times D_{\Omega}$, then $T_{h}{ }^{* \alpha} \alpha_{i}^{* \beta} \beta_{i}$ converges to $C * \alpha_{i} * \beta_{i}$, as well (Schwartz [1], II, p.53).

d) there remains to prove the last part of Theorem 1 . For $\mu \in D\left(L^{1}\right)$ and $T \in B^{\prime}$, noting that $F_{i} \in L^{\infty}$, we have:

$$
\left|<T_{0}(x+h), \mu(x)>\right| \leqq \sum_{i=0}^{2} \int_{R^{n}}\left|F_{i}(x+h) \Delta^{i k}{ }_{\mu}(x)\right| d x \leq \sum_{i=0}^{2} M_{i} \int_{R^{n}}\left|\Delta^{i k} \mu(x)\right| d x,
$$

where $M_{i}=\sup \left|F_{i}(x)\right|, x \in R^{n}$. Hence the set $\left\{T_{0}(x+h), h \in R^{n}\right\}$, is weakly bounded in $B^{\prime}$. Since $D$ is dense in $O\left(L^{1}\right)$, by the Banach-Steinhaus theorem the limit:

$$
\lim _{h \in \Gamma, h \rightarrow \infty}\left\langle T_{0}(x+h), \mu(x)>, \mu \in O\left(L^{1}\right),\right.
$$

exists, as well, and equals $\langle C, \mu\rangle$.

\section{ABELIAN THEOREMS FOR THE STIELTJES TRANSFORM}

THEOREM 2. Suppose that $T \in D^{\prime}$, the cone $\Gamma$ is with the nonempty interior and

i) $\mathrm{T}(\mathrm{x}+\mathrm{h}) \& \mathrm{c}(\mathrm{h}) \cdot \mathrm{U}(\mathrm{x}), \mathrm{h} \in \Gamma$ in $D^{\prime}$;

ii) For $a r>0$ and $s_{0} \in(C \backslash R)^{n}$ the distribution $T(x) /\left(s_{0}+x\right)^{r}$ belongs to $B^{\prime}$;

iii) For the same $r$ and $s_{0}, c(h) /\left(s_{0}+x+h\right)^{r}$ converges to $c_{1} \neq 0$ when $h \in \Gamma, h \rightarrow \infty$ and $x$ belongs to any compact set in $R^{n}$.

Then $T$ has the $S_{\rho}$-transform for all $\rho>r, S_{\rho}(T)(s)=\left\langle T(x) /\left(s_{0}+x\right)^{r}\right.$, $\left(s_{0}+x\right)^{r} /(s+x)^{\rho+e}>$ and

$$
\lim _{h \in \Gamma, h \rightarrow \infty} S_{\rho}(T)(s-x) / c(h)=0, \rho>r .
$$

PROOF. From supposition iii) it follows that the limit distribution $U=C$. Namely, for a $y \in R^{\mathrm{n}}$, x belonging to a compact set in $R^{\mathrm{n}}$ and $s_{0} \in(C \backslash R)^{\mathrm{n}}$ from the relation:

$$
\frac{c(h+y)}{c(h)}=\frac{c(h+y)}{\left(s_{0}+x+h+y\right)^{r}} \frac{\left(s_{0}+x+h\right)^{r}}{c(h)} \frac{\left(s_{0}+x+h+y\right)^{r}}{\left(s_{0}+x+h\right)^{r}}
$$

and from the Lemma 1 with the Remark after Lemma 1 , we have $\lim c(h+y) / c(h)=1$. Now, relation $(2.6)$ gives $U=C$. 
By Theorem X from [1], I, p. 72 (see also Pilipović and Stanković [2]), for a $\zeta \in$ $\epsilon$ it follows the existance of the limit:

$$
\begin{gathered}
\lim _{h \in \Gamma, h \rightarrow \infty}\left\langle T(x+h) /\left(s_{0}+x+h\right)^{r}, \zeta(x)\right\rangle=\lim _{h \in \Gamma, h \rightarrow \infty}\left\langle\frac{T(x+h)}{c(h)}, \frac{c(h)}{\left(s_{0}+x+h\right)^{r}} \zeta(x)\right\rangle= \\
=\left\langle C_{1}, \zeta(x)\right\rangle .
\end{gathered}
$$

In such a way we proved that the distribution $T(x) /\left(s_{0}+x\right)^{r}, s_{0} \in(C \backslash R)^{n}$, has the same properties as the distribution $T_{0}$ from Theorem 1 and we can use the assertion of this theorem.

We shall prove, now, the existence of the $S_{\rho}$-transform for $\rho>r$.

Since $\left|D^{k} \eta_{\omega}(x)\right| \leq C_{k} \omega^{(-k \cdot e)}, k \geqq 0$, where $C_{k}$ do not depend on $\omega$, the set of functions $\eta_{\omega}(x)(s+x)^{r-\rho-e}$ converges to $(s+x)^{r-\rho-e}, \rho>r$, in $D\left(L^{1}\right)$, when $\omega \rightarrow \infty$. Moreover, $\left(s_{0}+x\right)^{r} /(s+x)^{r}$, for $s_{0}, s \in(C \backslash R)^{n}$ belongs to $D\left(L^{\infty}\right)$; consequently

$$
\begin{gathered}
S_{\rho}(T)(s)=\lim _{\omega \rightarrow \infty}\left\langle T(x), \eta_{\omega}(x)(s+x)^{-\rho-e}\right\rangle=\lim _{\omega \rightarrow \infty}\left\langle\frac{T(x)}{\left(s_{0}+x\right)^{r}} \frac{\left(s_{0}+x\right)^{r}}{(s+x)^{r}}, \eta_{\omega}(x)(s+x)^{r-\rho-e}\right\rangle= \\
=\left\langle T(x) /\left(s_{0}+x\right)^{r},\left(s_{0}+x\right)^{r}(s+x)^{-\rho-e}>, \rho>r .\right.
\end{gathered}
$$

We have seen that the distribution $T(x) /\left(s_{0}+x\right)^{r}$ satisfies the conditions of Theorem 1 , therefore

$$
\begin{gathered}
\frac{1}{c(h)} S_{\rho}(T)(s-h)=\frac{1}{c(h)}<\frac{T(x+h)}{\left(s_{0}+x+h\right)^{r}},\left(s_{0}+x+h\right)^{r}(s+x)^{-\rho-e}>= \\
=\sum_{i=0}^{2} \frac{(-1)^{i(k \cdot e)}}{c(h)} \int_{R^{n}} F_{i}(x+h) \Delta^{i k} \frac{\left(s_{0}+x+h\right)^{r}}{(s+x)^{\rho+e}} d x .
\end{gathered}
$$

The expression $\Delta^{i k}\left[\left(s_{0}+x+h\right)^{r}(s+x)^{-\rho-e}\right]$ is given by the finite sum of elements which have the following form:

$$
H_{j, p}=C_{j, p}\left(s_{0}+x+h\right)^{r-j+p}(s+x)^{-\rho-e-p}, j \geq p \geq 0 .
$$

We shall analyse $H_{j, p} / c(h)$ when $x \in R^{n}$ and $h \in \Gamma$.

First we prove two inequalities:

$$
\begin{aligned}
& \left|\left(s_{0}+x+h\right)^{r}\right|=\prod_{i=1}^{n}\left|s_{0, i}+x_{i}+h_{i}\right|^{r_{i}} \leqq \prod_{i=1}^{n}\left(\left|s_{0, i}+h_{i}\right|+1\right)^{r_{i}}\left(\left|x_{i}\right|+1\right)^{r_{i}} \leqq \\
& \leq \prod_{i=1}^{n}\left|s_{0, i}+h_{i}\right|^{r_{i}}\left(1+\frac{1}{\left|\operatorname{Im} s_{0, i}\right|}\right)^{r_{i}} \prod_{i=1}^{n}\left(\left|x_{i}\right|+1\right)^{r_{i}} \leq \\
& \leq C_{r}\left|\left(s_{0}+h\right)^{r} / c(h)\right| \prod_{i=1}^{n}\left(\left|x_{i}\right|+1\right)^{r_{i}} \text {; } \\
& \left|\left(s_{0}+x+h\right)^{p-j}\right|=\prod_{i=1}^{n}\left|s_{0, i}+x_{i}+h_{i}\right|^{p_{i}-j_{i}} \leqq \prod_{i=1}^{n}\left|\operatorname{Im} s_{0, i}\right|^{p_{i}-j_{i}}=c_{p, j}^{\prime} .
\end{aligned}
$$

Now

$$
\left|H_{j, p}\right| / c(h) \leq c_{j, p} C_{j, p}^{\prime} C_{r}\left|\frac{\left(s_{0}+h\right)^{r}}{c(h)}\right| \prod_{i=1}^{n} \frac{\left(\left|x_{i}\right|+1\right)^{r_{i}}}{\left(s_{i}+x_{i}\right)^{\rho_{i}+p_{i}+1}}, \quad p \geq 0 .
$$

This inequality shows that $\left|\mathrm{H}_{j, p}\right| / c(h)$ is bounded by a function which belongs to $L^{1}$, when $h \in \Gamma$. Since $F_{i}(x+h)$ are bounded, as we 11 , when $x \in R^{n}$ and $h \in \Gamma$, we can use the Lebesgue theorem for the integral in (5.5) to obtain that $S_{\rho}(T)(s-h) / c(h)$ tends to zero when $\mathrm{h} \in \Gamma, \mathrm{h} \rightarrow \infty$. 
The next theorem presents more precisely how $S_{\rho}(T)(s-h)$ tends to zero when $h \in r_{2}$, $h \rightarrow \infty . \Gamma_{2}$ is defined in the Remark after Lemma 1 . We have seen that $\left|h_{i}\right| \rightarrow \infty, i=1, \ldots$ $\ldots, n$, if $h \rightarrow \infty, h \in \Gamma_{2}$.

THEOREM 3. Let $T \in O^{\prime}$ and $s_{0} \equiv\left(s_{0,1} \cdots s_{0, n}\right) \in(C \backslash R)^{n}$. We suppose:

i) $\left(s_{0, j}+x_{j}+h_{j}\right) T(x+h) \stackrel{s}{\sim} 1 \cdot C, h \in \Gamma$ in $D^{\prime} ; 1 \leq j \leq n$

ii) $\left(s_{0, j}+x_{j}\right) T(x) \in B^{\prime}$.

Then, for $\rho>0$ and $\Gamma$ convex cone, $\stackrel{\Gamma}{\Gamma} \neq \emptyset$

$$
\lim _{h \in \Gamma_{2}, h \rightarrow \infty} h_{j} S_{\rho}(T)(s-h)=0
$$

PROOF. We can write:

$$
\begin{gathered}
s_{\rho}\left(\left(s_{0, j}+x_{j}\right) T(x)\right)(s-h)=\left\langle\left(s_{0, j}+x_{j}+h_{j}\right) T(x+h),(s+x)^{-\rho-e}\right\rangle= \\
=\left\langle T(x+h),(s+x)^{-\rho-e^{\prime}}\right\rangle+\left(s_{0, j}-s_{j}+h_{j}\right)\left\langle T(x+h)(s+x)^{-\rho-e}\right\rangle ; e^{\prime}=\left(e_{1}^{\prime} \ldots e_{n}^{\prime}\right),
\end{gathered}
$$

where $e_{i}^{\prime}=1, i \neq j$ and $e_{j}^{\prime}=0$. Hence

$$
s_{\rho}(T)(s-h)=\left(s_{0, j}-s_{j}+h_{j}\right)^{-1}\left[s_{\rho}\left(\left(s_{0, j}+x_{j}\right) T(x)\right)(s-h)-\left\langle T(x+h),(s+x)^{-\rho-e^{\prime}}\right\rangle\right]
$$

First, we shall prove that $S_{\rho}\left(\left(s_{0, j}+x_{j}\right) T(x)\right)(s-h) \rightarrow 0$ when $h \in \Gamma_{2}, h \rightarrow \infty$. By Theorem 1 it follows:

$$
\lim _{h \in \Gamma}, h \rightarrow \infty S_{\rho}\left(\left(s_{0, j}+x_{j}\right) T(x)\right)(s-h)=\left\langle C,(s+x)^{-\rho-e}\right\rangle=0
$$

It remains to prove that $\left\langle T(x+h),(s+x)^{-\rho-e^{\prime}}>\rightarrow 0\right.$ when $h \in \Gamma_{2}, h \rightarrow \infty$, as well. By Theorem 1 we know that our distribution $T$ has the form

$$
T(x)=\left(s_{0, j}+x_{j}\right)^{-1} \sum_{i=0}^{2} \Delta \Delta_{i} F_{i}(x)
$$

where $F_{i}(x)$ are bounded functions when $x \in R^{n}$. Using this form of $T$, we have:

$$
\left\langle\mathrm{T}(\mathrm{x}+\mathrm{h}),(\mathrm{s}+\mathrm{x})^{-\rho-\mathrm{e}^{\prime}}\right\rangle=\sum_{i=0}^{2}(-1)^{\mathrm{i}(\mathrm{k} \cdot \mathrm{e})}\left\langle\mathrm{F}_{\mathrm{i}}(\mathrm{x}+\mathrm{h}), \Delta^{i k}\left(s_{0, j}+\mathrm{x}_{j}+\mathrm{h}_{j}\right)^{-1}(s+\mathrm{x})^{-\rho-e^{\prime}}\right\rangle
$$

The second part of relation (5.15) consists in fact of a sum of integrals

$$
\int_{R^{n}} F_{i}(x+h)\left(s_{0, j}+x_{j}+h_{j}\right)^{-m}(s+x)^{-\rho-\alpha} d x
$$

where $m \geq 1 ; \alpha_{j} \geq 0$ and $\alpha_{i} \geq 1, i \neq j$. All of these integrals tend to zero when $h \in \Gamma_{2}$, $h \rightarrow \infty$. We shall prove only the case: $m=1, \alpha_{j}=0$. In other cases it is trivial.

Let us consider the integral

$$
\int_{-\infty}^{\infty} F_{i}(x+h)\left(s_{0, j}+x_{j}+h_{j}\right)^{-1}\left(s_{j}+x_{j}\right)^{-\rho} j_{d x_{j}}
$$

for $x_{k} \in R, k \neq j$ and $h \in \Gamma_{2}$. The function in $x_{j} F_{i}(x+h)\left(s_{0, j}+x_{j}+h_{j}\right)^{-1}$ belongs to $D^{\prime}\left(L^{v}\right)$ for every $v>1$. Let $p$ be such that $p \rho_{j}>1$ and $\frac{1}{p}-\frac{1}{v}-1 \geq 0$. Then, for $x_{i} \in R, i \neq j$, integral (5.17) is bounded by a function in $h$ belonging to $O\left(L^{u}\right), \frac{1}{u}=\frac{1}{v}+\frac{1}{p}-1$ (Schwartz [1], II, p. 60).

Now, we have to prove that we can find $p$, satisfying our conditions, and $v>1$, such that the number $u$ remains in the interval $1 \leq u<\infty$. Then, we have only to use the 
property of a $\zeta \in O\left(L^{\mathrm{u}}\right), 1 \leqq u<\infty$; namely: $\zeta(\mathrm{x}) \rightarrow 0,\|x\| \rightarrow \infty$ (Schwartz [1], II, p. 55) and it will follow that integral (5.16) tend to zero when $h \in \Gamma_{2}, h \rightarrow \infty$. Therefore we shall analyse two cases.

In case $\rho_{j} \geqq 1$, we can take $\frac{1}{p}=1-\frac{1}{2 v}$, hence $u=2 v$.

In case $\rho_{j} \geqq 1$, we can take $\frac{1}{p}=1-\frac{1}{2 v}$, hence $\frac{1}{v}+\frac{1}{p}-1 \geqq 0$, we have $1>\frac{1}{v} \geqq 1-\frac{1}{p}>1-\rho_{j}$, or $1<v<\left(1-\rho_{j}\right)^{-1}$. For such an $v$ the number $1 / u$ is strictly positive, hence $1 \leqq u<\infty$.

The next example shows that Theorem 3 cannot be proved for $\rho=0$

$$
\int_{0}^{\infty} \frac{d x}{(x+a)(s+x)}=(a-s)^{-1} \ln \left(\frac{a}{s}\right), a, s>0 \text {. }
$$

There arises an another question: If we know that $\left(s_{0, j}+x_{j}\right) r^{r} T(x) \in B^{\prime}$ for a $r>1$, is it true that $S_{\rho}(T)(s-h)=o\left(h_{j}^{-r}\right), h \in r_{2}, h \rightarrow \infty$ ? The answer is in general negative. This shows the following integral:

$$
\int_{0}^{\infty} e^{-t} \frac{d t}{(s+t)^{v}}=s^{-v / 2} \cdot \exp (s / 2) w_{-v / 2,(1-v) / 2}(s) \sim s^{-\nu}, \quad s>0, s \rightarrow \infty,
$$

where $W_{\nu, \mu}$ is the Whittaker function.

ACKNOWLEDGEMENT. This material is based on work supported by the U.S.-Yugoslavia Joint Fund For Scientific and Technological Cooperation, in cooperation with the NSF under Grant (JF) 838.

\section{REFERENCES}

1. SCHWARTZ, L. Théorie des distributions, T. I-II, Hermann, Paris, 1950-1951.

2. PIIPOVIĆ, S. and STANKOVIĆ, B. S-asymptotic of a distribution, Pliska Stud. Math. Bulgar., Vo1 10, (1989), 147-156.

3. STANKOVIĆ, B. S-asymptotic expansion of distributions, Internat. J. Math. Math. Sci. Vol. 11, No. 3 (1988), 449-456.

4. VLADIMIROV, V.S. Generalized Functions in Mathematical Physics, MIR Publisher, Moscow, 1979.

5. LAVOINE, J. and MISRA, O.P. Théorèmes Abéliens pour la transformation de Stieltjes des distributions, C. R. Acad. Sc. Paris Sér. I Math. T 279 (1974), 99-102.

6. LAVOINE, J. and MISRA, O.P. Sur la transformation de Stieltjes des distributions et son inversion au moyen de la transformation de Laplace, C. R. Acad. Sc. Paris Sér. I Math. T. 290 (1980), 139-142.

7. ZEMANIAN, A.H. Generalized integral transformations, Intersciences, New York, 1986.

8. PANDEY, J.N. On the Stieltjes transform of generalized functions, Math. Proc. Cambridge Philos. Soc., 71 (1971), 85-96.

9. BREMERMANN, H. Distributions, Complex Variables and Fourier Transforms, AddisonWesley, Reading, Mass, 1965.

10. PILIPOVIĆ, S. and STANKOVIĆ, B. Abelian theorem for the distributional Stieltjes transform, Z. Ana1. Anwendungen Bd. 6(4), (1978), 341-349. 


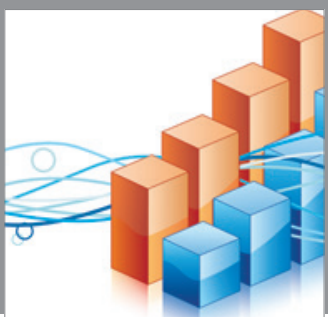

Advances in

Operations Research

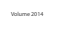

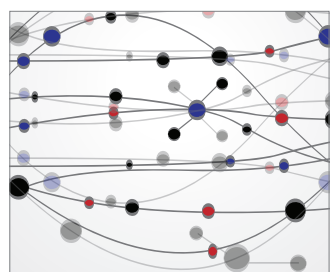

\section{The Scientific} World Journal
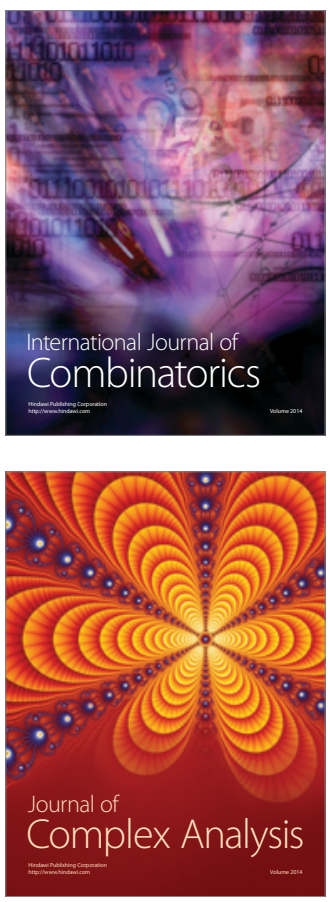

International Journal of

Mathematics and

Mathematical

Sciences
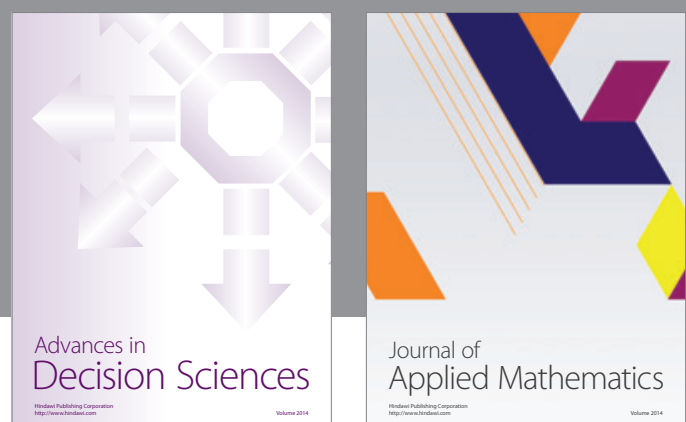

Journal of

Applied Mathematics
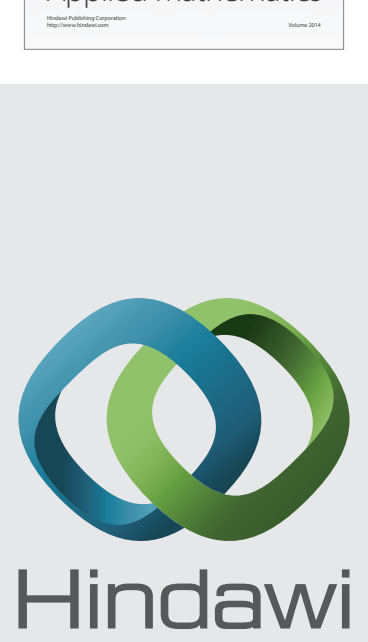

Submit your manuscripts at http://www.hindawi.com
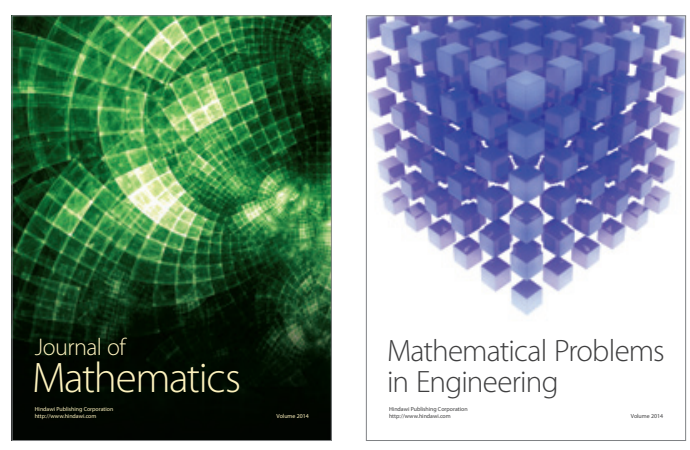

Mathematical Problems in Engineering
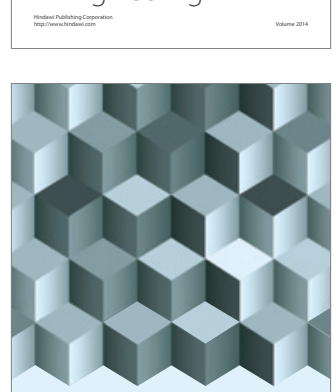

Journal of

Function Spaces
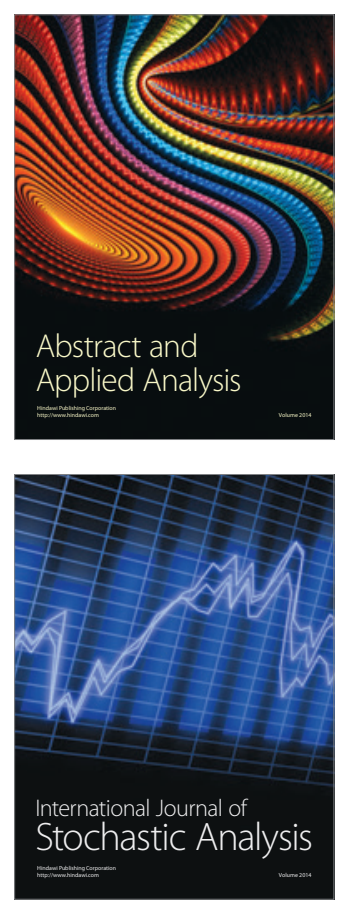

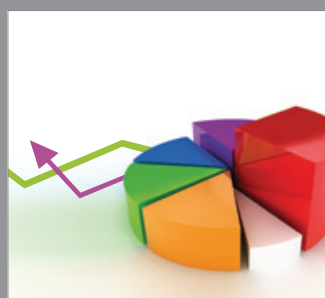

ournal of

Probability and Statistics

Promensencen
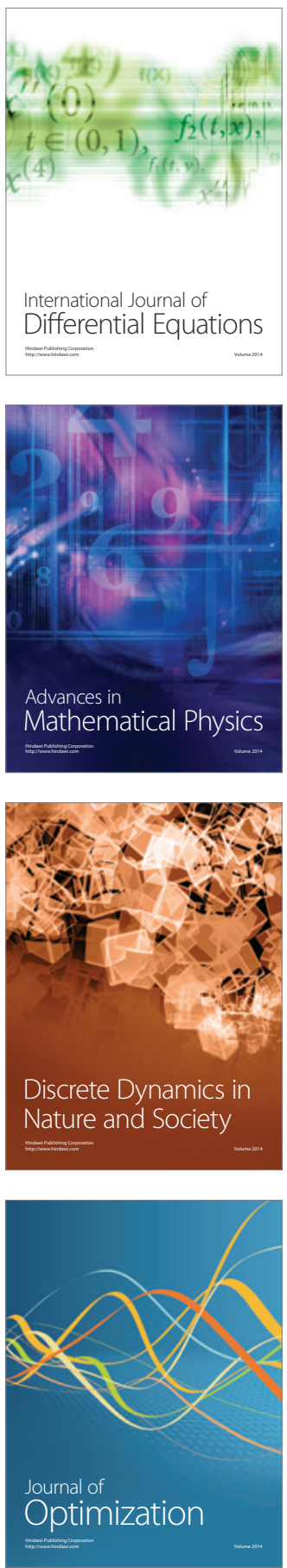